\title{
Uma análise bibliométrica sobre componentes biomecânicos de titânio ligados a elementos betagênicos aplicados em áreas biomédicas e de saúde
}

\author{
A bibliometric analysis of the biomechanical components of titanium linked to betagenic elements \\ applied in biomedical and health areas
}

Un análisis bibliométrico de componentes biomecánicos del titanio ligados a elementos beta aplicados en áreas biomédicas y de salud

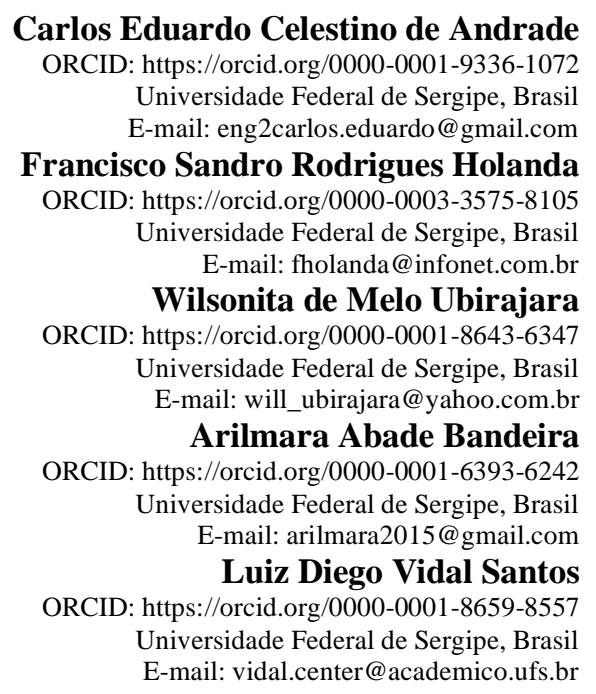

\section{Resumo}

A produção de inovações tecnológicas a partir de materiais metálicos biomédicos exibe extraordinário valor para a reconstrução de tecidos estruturais que falharam e assim, atualmente, ligas de titânio com elementos betagênicos surgem como opção, principalmente para minimizar as limitações quanto ao módulo de elasticidade bem como, a citotoxidade das ligas amplamente comercializadas. O objetivo deste estudo foi produzir indicadores científicos e tecnológicos para delinear o ambiente de desenvolvimento tecnológico das ligas de titânio do tipo beta para o período de 2016 a 2020 , através da consulta no repositório de dados Scopus e na World Intelectual Property Organization - WIPO. Os resultados mostraram que a China, o Brasil, a Índia, a Austrália e a Alemanha são os países com maiores números de publicações de artigos acadêmicos na temática. Entretanto, a China e os Estados Unidos da América são os países que mais depositaram patentes, correspondendo cada um a 27\% dos depósitos.

Palavras-chave: Rigidez; Inovação; Prospecção.

\begin{abstract}
The production of technological innovations from biomedical metallic materials exhibits extraordinary value for the reconstruction of structural tissues that have failed and thus, currently, titanium alloys with betagenic elements appear as an option, mainly to minimize the limitations regarding to elasticity module not only, but also, the cytotoxicity of widely traded alloys. The objective of this study was to perform scientific and technological indicators to outline the technological development environment of the type titanium alloys for the period from 2016 to 2020 , by consulting the Scopus Elsevier data repository and the World Intellectual Property Organization - WIPO. The results showed that China, Brazil, India, Australia and Germany are the countries with the highest numbers of academic article publications on this subject. However, China and the United States of America are the countries that most deposited patent orders, each accounting for $27 \%$ of deposits.
\end{abstract}

Keywords: Rigidity; Innovation; Prospection. 


\section{Resumen}

La producción de innovaciones tecnológicas a partir de materiales metálicos biomédicos exhibe un valor extraordinario para la reconstrucción de tejidos estructurales que han fallado y así, en la actualidad, las aleaciones de titanio con elementos betagénicos aparecen como una opción, principalmente para minimizar las limitaciones en cuanto al módulo de elasticidad, así como, la citotoxicidad de aleaciones ampliamente comercializadas. El objetivo de este estudio fue producir indicadores científicos y tecnológicos para delinear el entorno de desarrollo tecnológico de las aleaciones de titanio tipo beta para el período de 2016 a 2020, consultando el repositorio de datos de Scopus y la Organización Mundial de la Propiedad Intelectual - OMPI. Los resultados mostraron que China, Brasil, India, Australia y Alemania son los países con mayor número de publicaciones de artículos académicos sobre el tema. Sin embargo, China y los Estados Unidos de América son los países que han presentado más patentes, cada uno con el 27\% de los depósitos.

Palabras clave: Rigidez; Innovación; Prospección.

\section{Introdução}

A necessidade de uma perfeita reconstituição de seções ósseas comprometidas após acidentes ou doenças evoluiu para o desenvolvimento de implantes estruturais baseados em ligas de titânio (Taddei, Henriques, Silva, Cairo, \& Bottino, 2007). A experiência do uso e o progresso das pesquisas trouxeram a percepção de problemas associados aos implantes ortopédicos como o afrouxamento da prótese no osso em presença de uma matriz ortopédica baseada em elementos com potencial citotóxico o que devido a interação de superfície pode provocar o desgaste, eliminando elementos como o vanádio e o alumínio causando uma série de problemas ao portador do implante. Uma possibilidade estudada para contornar este inconveniente consiste na adição de um ou mais elementos químicos sem potencial tóxico (Almanza, Pérez, Rodríguez, \& Murr, 2017). Atualmente tem sido observado na literatura especializada (Lee et al., 2020; Nunes et al., 2020; Wu et al., 2020; Xu et al., 2020), movimento que denota o forte interesse em ligas de titânio do tipo beta para serem aplicadas em áreas biomédicas e de saúde. Essas ligas, têm atraído atenção devido as suas propriedades de elevada resistência à degradação, e boa relação força-peso, características já encontradas em ligas dos tipos $\alpha$ e $\alpha+\beta$ amplamente comercializadas (Almanza et al., 2017; Murillo et al., 2019), mas com os benefícios de apresentarem biocompatibilidade, baixo Módulo de Elasticidade - ME e nenhum potencial citotoxidade.

Uma elevada diferença entre o ME do implante metálico e do tecido ósseo oportuniza um acúmulo de carga no implante, causando fragilização mecânica do osso em sua volta (Niinomi, Nakai, \& Hieda, 2012). Além disso, os elementos betagênicos que integram a liga apresentam baixa capacidade de promover alteração metabólica nas células quando em uso (Taddei et al., 2007). Em geral, estas propriedades são critérios prioritários durante o procedimento de seleção do material a ser utilizado em aplicações biomecânicas e são constituídos, basicamente, por uma matriz de titânio e por elementos betagênicos, como Mo, $\mathrm{Nb}$, Ta, Sn e Zr.

$\mathrm{O}$ ato de tornar público uma pesquisa, como parte central da pesquisa científica, é um importante indicador de contribuição para o meio acadêmico. A análise bibliométrica é utilizada para avaliar qualitativa e quantitativamente tendências na atividade de pesquisa por meio destas publicações ao longo do tempo (Pu, Lyu, \& Su, 2016). Este tipo de análise quando bem planejada, contando com bons descritores e utilizando-se de bases de dados de literatura robustos e fiéis, fornece ao pesquisador uma maneira de aproveitar o desenvolvimento em um determinado campo e comparar as contribuições de estudiosos, periódicos, institutos e países, a fim de que possa refutar seus dados ou corroborar seus achados que muitas vezes não se explicam com apenas dados matemáticos, tornando os experimentos mais válidos (Souza Neto et al., 2019).

As patentes, assim como as publicações científicas, representam instrumentos jurídicos diretos e indiretos de proteção do invento resultante de um esforço de pesquisa dos institutos de pesquisa, e de pesquisadores no desenvolvimento de processos tecnológicos e novos produtos ( $\mathrm{Li}$ et al., 2020). Isto torna um investimento mais seguro, com potencial fonte rentável e legítimo se prevenindo do comportamento desleal que pode surgir da concorrência, evitando a comercialização de cópia não permitida, aspecto que desrespeita o esforço realizado pelos detentores originais e os gastos empregados no desenvolvimento (Marques \& Marques, 2017). 
Os documentos que compõem os pedidos de patente são constituídos por partes: relatório descritivo, reivindicações e resumo (INPI, 2017). Por sua natureza redacional, deve descrever o objetivo, funcionalidades e reivindicações das proteções solicitadas, mostrando-se um texto capaz de demonstrar quais os aspectos da invenção e quais os pontos inéditos que trazem esta novidade industrial, expressando desta forma possíveis inovações (WIPO, 2018). Tais documentos podem ser explorados por outros inventores e pesquisadores a fim de compreender o estado da arte de determinadas pesquisas e áreas de invenções. Mostrase importante a busca da compreensão atual das propriedades intelectuais e inovações tecnológicas à luz das áreas derivadas dos contextos dos componentes biomecânicos, a fim de que seja possível compreender o contexto e caminho do desenvolvimento tecnológico (Mazieri, Quoniam, \& Santos, 2016).

À medida que o número de idosos que compõem a população mundial aumenta, uma vez que estes apresentam um risco maior de necessitar que o tecido duro seja reconstruído, cresce também a demanda por biomateriais metálicos. Porém, a biocompatibilidade biológica e mecânica dos biomateriais metálicos ainda requerem muitas melhorias (Niinomi et al.,2012). Em tempo, a maioria dos implantes tem sido realizada com o uso de biomateriais metálicos, que são extremamente importantes para a reconstrução dos tecidos, especialmente tecidos duros.

Dado esta perspectiva, é pertinente convir que inovações tecnológicas focadas em desempenho estrutural para implantes ortopédicos têm sido amplamente pesquisadas (Lee et al., 2020; Vieira Nunes et al., 2020; Wu et al., 2020; Xu et al., 2020). Ocorre que, até então, é relevante a realização de um estudo capaz de mapear tendências e sinais de mudanças para estabelecer conjecturas mercadológicas necessárias para estas ligas. Ocorre que, até então, é relevante a realização de um estudo capaz de mapear tendências e sinais de mudanças para estabelecer conjecturas mercadológicas necessárias para estas ligas.

Torna-se relevante, diante deste cenário, prognosticar quais rumos o desenvolvimento tecnológico está seguindo, por meio da identificação do que está sendo gerado de conhecimento específico a respeito desta temática, detectando o nível de registro de manuscritos, não só, mas também de patentes, sendo este um relevante instrumento, que garante a exploração de suas inovações de maneira exclusiva por determinado período de tempo. O levantamento de patentes permite, entre outros, não só identificar soluções técnicas como também o desenvolvimento tecnológico nas diversas instituições e países permitindo que surjam inovações em diversos campos de exploração e teste, para novos materiais e equipamentos tornando-se potencialmente a solução e/ou amenização de diversas problemáticas desta área.

Portanto, o objetivo deste estudo foi produzir indicadores científicos e tecnológicos para delinear o ambiente de desenvolvimento tecnológico das ligas de titânio do tipo do tipo beta para o período de 2016 a 2020, através da consulta no repositório de dados Scopus Elsevier e na World Intelectual Property Organization - WIPO.

\section{Metodologia}

Foi escolhido o repositório de dados Scopus Elsevier para o mapeamento dos indicadores científicos e determinação da estrutura lógica de busca para os indicadores tecnológicos, com o auxílio da ferramenta de acesso gratuito VOSviewer, realizado na base de dados de patentes da WIPO. Este procedimento metodológico utilizado foi o qualitativo, haja vista, a execução da análise hermenêutica para interpretação dos dados objeto de estudo, conforme cita Pereira et al. (2018) e foi dividido em duas etapas:

Etapa 1: Inicialmente buscou-se o estabelecimento dos descritores a serem utilizados, sob uma ótica ampla, de maneira a estarem intrinsecamente relacionados com o objetivo da pesquisa no repositório de dados Scopus. Assim, foram utilizados os descritores ("beta type" AND "titanium alloy" AND "orthopedic" AND "implant">2016) e realizada a procura para leitura de títulos, resumos e análise de palavras-chave, selecionando-se publicações científicas recentes dos últimos 5 anos para construção de mapas de visualização de termos aplicado para dados de co-ocorrência e exportados os arquivos segundo a extensão CSV Excel. 
Etapa 2: A visualização de sobreposição de termos possibilitou definir a estrutura lógica para a busca dos indicadores tecnológicos na base de dados WIPO: (titanium alloys AND biocompatibility AND niobium alloys). Foi escolhido a forma de pesquisa avançada com critério (página de cobertura) e refinamento para as patentes publicadas para o período 2016 - 2020.

Este tipo de estudo possibilita o levantamento das tecnologias existentes, identificando o estágio de maturidade da tecnologia em questão e sua inserção na sociedade, bem como os aspectos relativos a tecnologias concorrentes (Quintella, Meira, Kamei, Tanajura \& da Silva, 2011). Por se tratar de uma prospecção científica/tecnológica, o trabalho é classificado como uma pesquisa do tipo exploratória, que "[...] tem como finalidade proporcionar mais informações sobre o assunto que vamos investigar, possibilitando sua definição e delineamento" (Prodanov \& Freitas, 2013, p. 51). Neste contexto, o presente artigo pode também servir como instrumento de estudo de anterioridade para aqueles que pretendam trabalhar com o tema abordado.

\section{Resultados e Discussão}

A base de dados Scopus localizou 23 trabalhos científicos sobre o tema proposto, enquanto a busca de patente na plataforma WIPO retornou um total de 15 patentes publicadas, sendo este o universo da prospecção sobre o qual serão realizadas as análises.

Quanto à distribuição das publicações ao longo dos anos estudados, a Figura 1 mostra de forma geral, um número restrito de publicações acadêmicas 2017 (5), 2018 (5), 2019 (8) e 2020 (5). Essa distribuição pode ser interpretada, quando se é correlacionado aspectos de custos incorridos em toda cadeia produtiva do titânio e suas ligas, assim como, quando se relacionada a dinâmica de formação e a de atuação da pesquisa acadêmica (Tubella, 2019).

Nota-se, ainda, um incremento na quantidade de publicações para o ano de 2019, não só, mas, a impossibilidade de afirmar se o ano de 2020 apresentará queda do número de publicações em relação ao ano anterior, como pode sugerir a Figura 1 , uma vez que no momento e realização da busca falta ainda um trimestre para seu encerramento.

Figura 1. Distribuição da produção científica sobre desenvolvimento tecnológico das ligas de titânio.

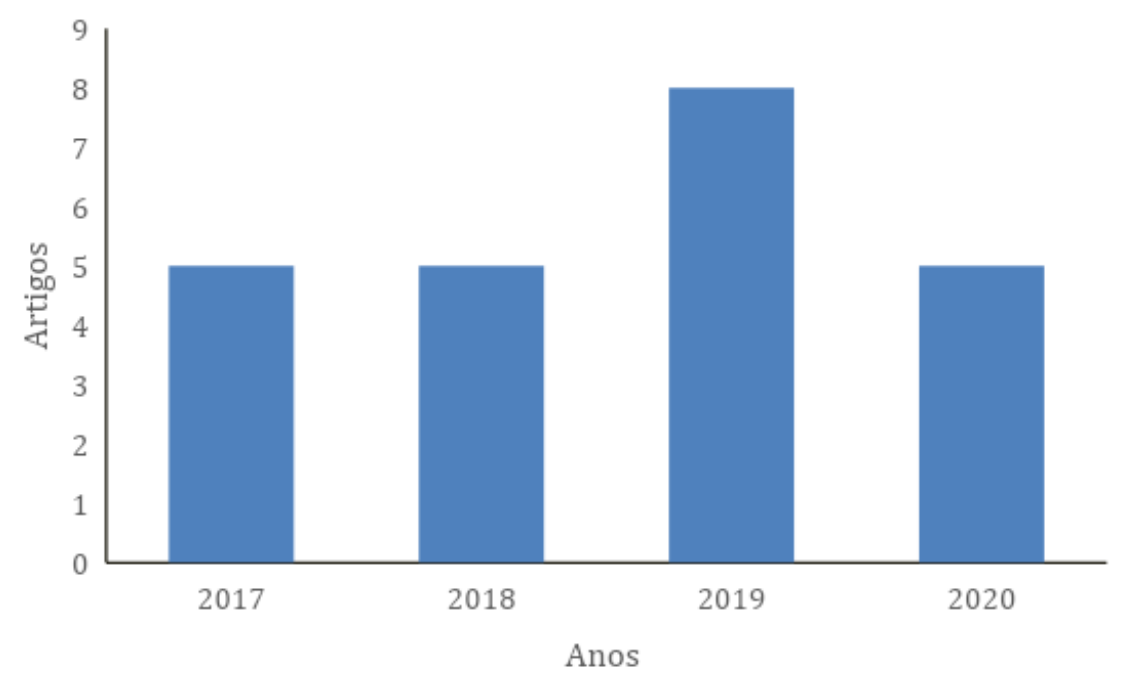

Fonte: Autores (2021).

No que concerne aos países envolvidos nas publicações, a Figura 2 apresenta a China com o maior número de publicações no período, com um total de 8 artigos, seguido pelo Brasil, com 6 artigos; Índia, com 4 artigos; e Austrália e Alemanha, ambas com 2 artigos publicados no período. 
Um fator importante a se observar é que na cultura atual o comportamento de crescimento de publicações de inovações resultantes de pesquisas acadêmicas tem aumentado em todo o mundo (Chevalier \& Conrozier, 2017; Kyvik \& Aksnes, 2015). Fontelo e Liu (2018), em um estudo que analisou o registro de publicações depositadas no banco de dados Pubmed no período de 1995 a 2015, verificaram que, dos países asiáticos, a China ficou em segundo lugar no total de publicações, mostrando que a cultura de publicações de resultados científicos sobre pesquisas médicas pela China é atualmente forte e pode impactar quantitativamente em diversos campos correlatos às pesquisas em saúde tais como, às áreas de estudos sobre os componentes biomecânicos de titânio ligados a elementos betagênicos.

Figura 2 - Produção científica sobre desenvolvimento tecnológico das ligas de titânio por país.

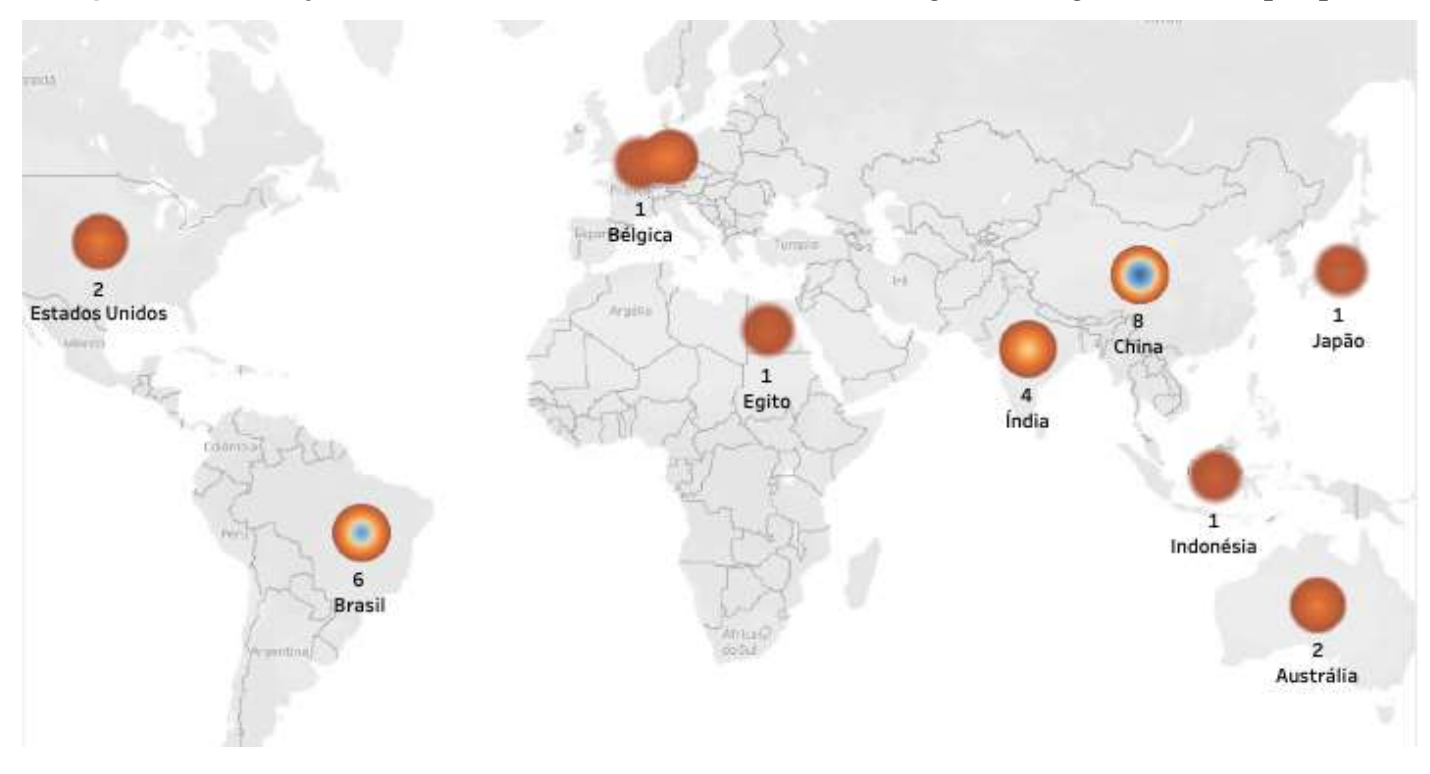

Fonte: Autores (2021)

Em consonância com os destaques de cada país, é possível observar na Figura 3 a presença predominante de instituições de ciência e tecnologia da China e do Brasil, sendo este último o país de origem das três das quatro principais instituições, a saber: UNESP-Universidade Estadual Paulista, Centro Universitário de Volta Redonda e Universidade Federal do Rio de Janeiro. As dez instituições apresentadas na Figura 3 são responsáveis por 69,5\% dos artigos da amostra obtida. 
Figura 3 - Produção científica por instituição sobre desenvolvimento tecnológico das ligas de titânio.

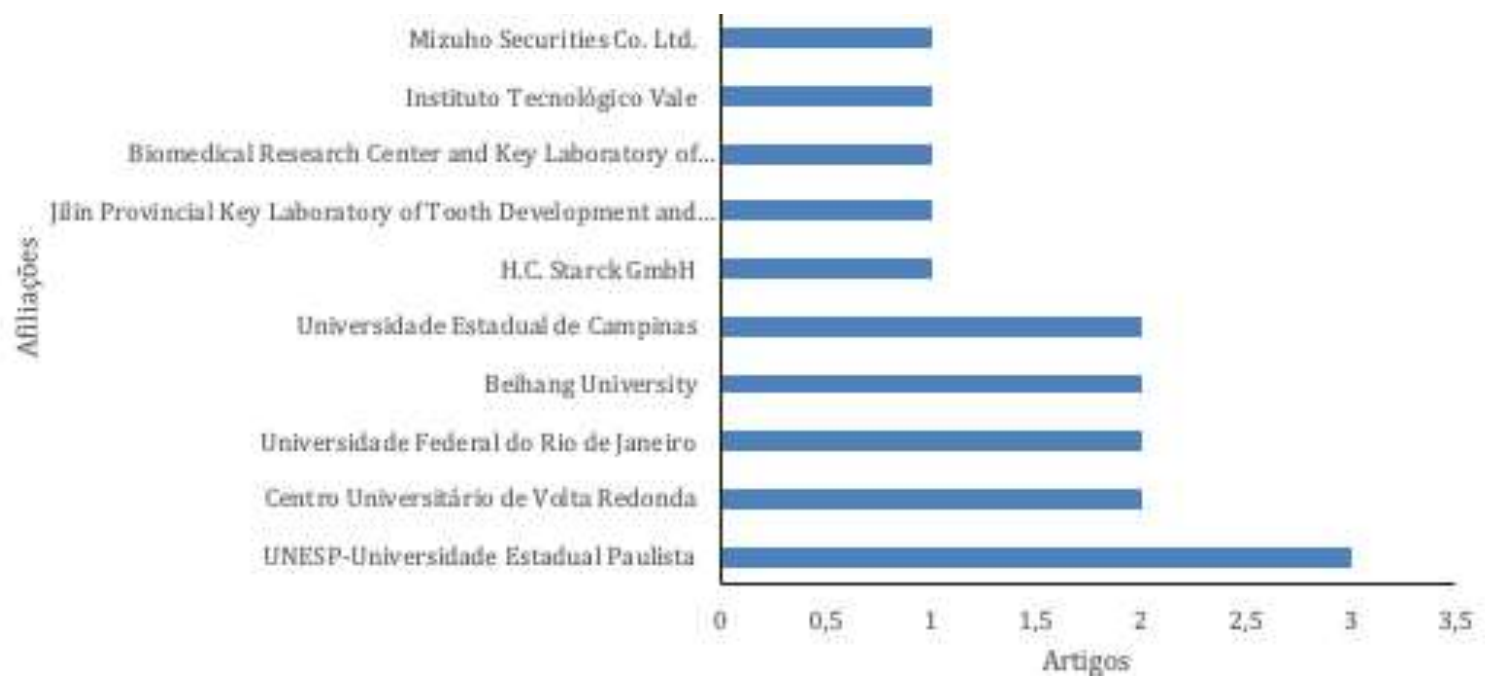

Fonte: Autores (2021).

Os resultados apresentados neste estudo demonstram que a maioria das publicações estão em revistas que abordam os temas relacionados a Biocompatibilidade e Bio-interações, corroborando com outros relacionados ao tema (Fontelo \& Liu, 2018; Teng, Chew, Gupta, \& Yeap, 2020; Xing, Zhao, Dong, \& Lin, 2018), que são: as interações material-biologia, comunicação química/morfológica/estrutural, mecanobiologia, sinalização e respostas biológicas, imunoengenharia, calcificação, revestimentos, corrosão e degradação de biomateriais e dispositivos, regulação biofísica das funções celulares.

Ademais, dos 23 artigos científicos encontrados na base Scopus, 56,5\% estão publicados em 10 periódicos, em que se destacam ACS Biomaterials Science And Engineering, Materials Science And Engineering C Materials Science Forum, conforme observado na Figura 4. Segundo Araújo (2006), o levantamento dos periódicos nos quais foram publicados a maior parte dos documentos atende à Lei de Bradford, que permite identificar o grupo de periódicos que publicam o maior número de artigos sobre o tema, formando um núcleo de periódicos de maior relevância para determinada área.

É válido observar que três dos dez periódicos contidos nesta lista apresentam boas pontuações no Journal Citation Report (JCR). Bioactive Materials (JCR 8.724), Journal of Biomedical Materials Research (JCR 3.652) e ACS Biomaterials Science \& Engineering (JCR 4.152) demostrando que, os temas aqui observados, apresentam altos níveis de busca relacionados ao tema assim como relevância atual. O JCR é um fator de impacto medido pela base estatística InCites da editora Clarivate Analytics (ABEC, 2017). Ele é um recurso que permite avaliar e comparar publicações científicas utilizando dados de citações extraídos de revistas acadêmicas e técnicas e o impacto destas na comunidade científica indexadas pela coleção principal da Web of Science (USP, 2020). 
Figura 4 - Publicações científicas sobre desenvolvimento tecnológico das ligas de titânio por periódicos.

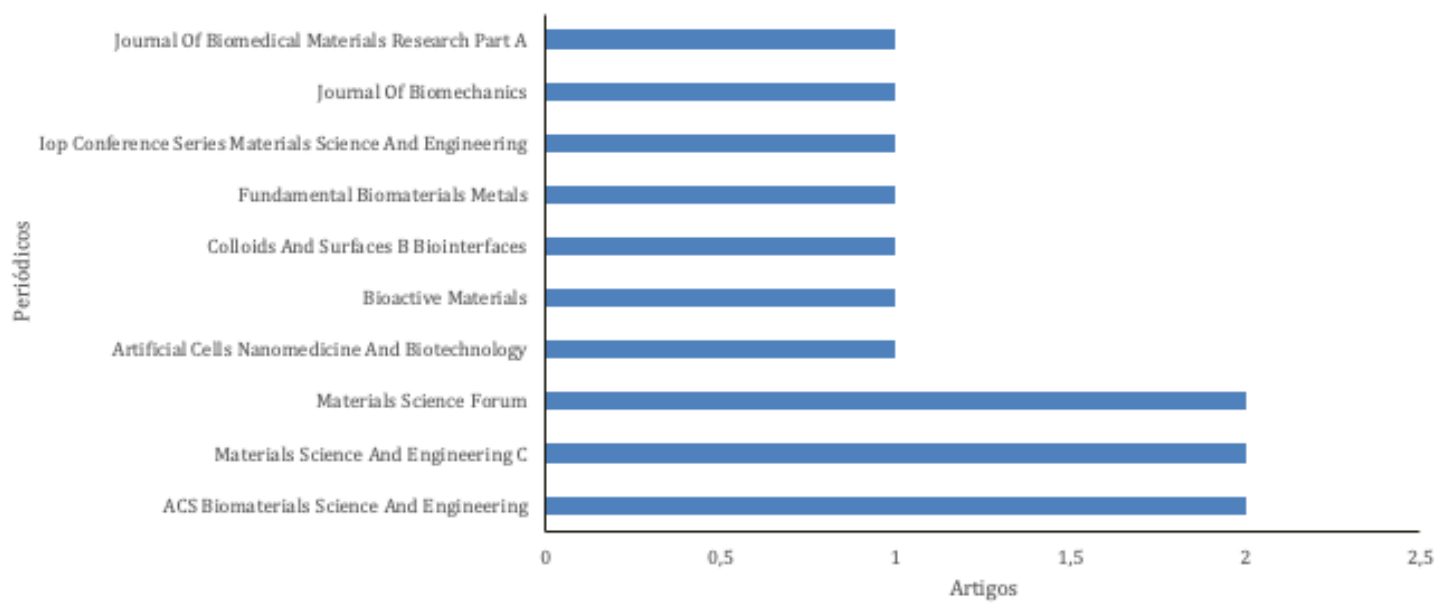

Fonte: Autores (2021)

No escopo da busca de patentes na base da WIPO, a Figura 5 apresenta os principais requerentes de patentes de liga de titânio, percebendo-se a presença de duas empresas, Smith \& Nephew Richards Inc. e Dalian Itriup Science \& Technology Development Co., Ltd., uma universidade, Beihang University, seis inventores independentes, Davidson James A, Hui Songxiao, Kovacs Paul, Lee Dong Geun, Lee Yong Tae, Mi Xujun, o que reflete o intenso crescimento e variabilidade do mercado de biomateriais, cujo impacto na melhoria da qualidade da vida humana é incontestável e cuja contribuição futura deve ser proeminente, tendo em vista a tendência bem estabelecida de envelhecimento populacional ao redor do mundo (Pires, Bierhalz, \& Moraes, 2015).

Figura 5 - Requerentes de patentes de ligas de titânio.

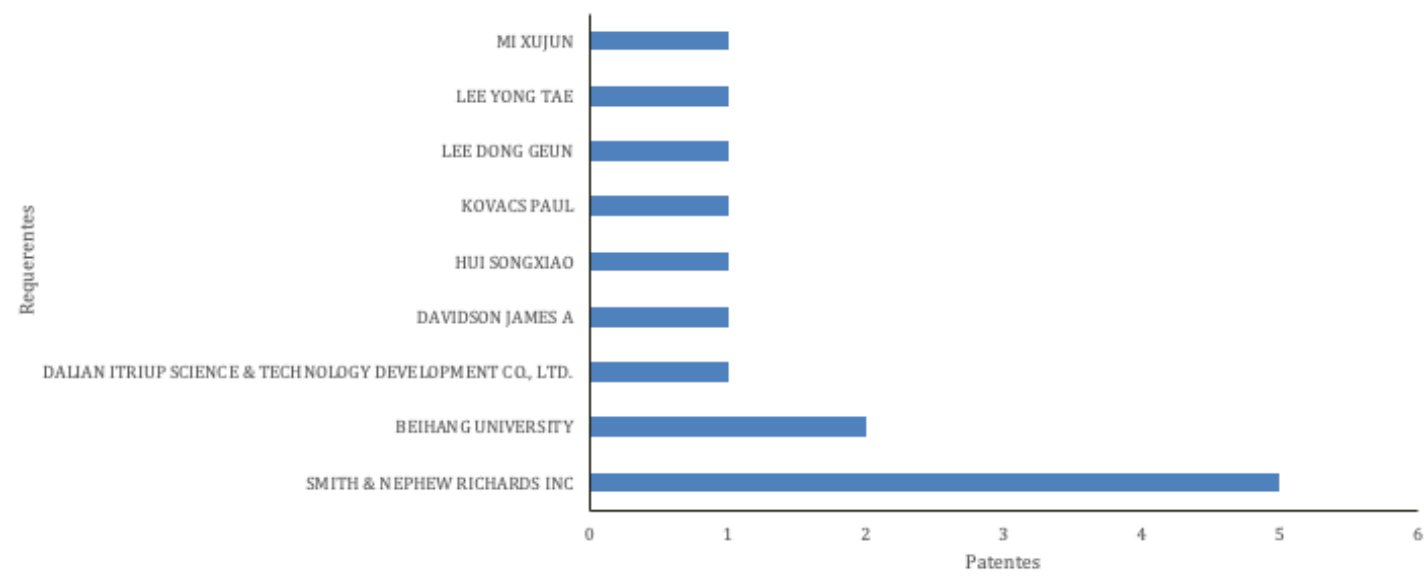

Fonte: Autores (2021).

Na Figura 6 observa-se os principais países em que foram depositadas as patentes de liga de titânio, sendo a China e os Estados Unidos da América os que mais depositaram pedidos, correspondendo cada um a 27\% dos pedidos. Comparando-se as informações com a Figura 5, percebe-se que os dois países de destaque correspondem aos principais países de origem da empresa, universidade ou inventor requerente da patente. 


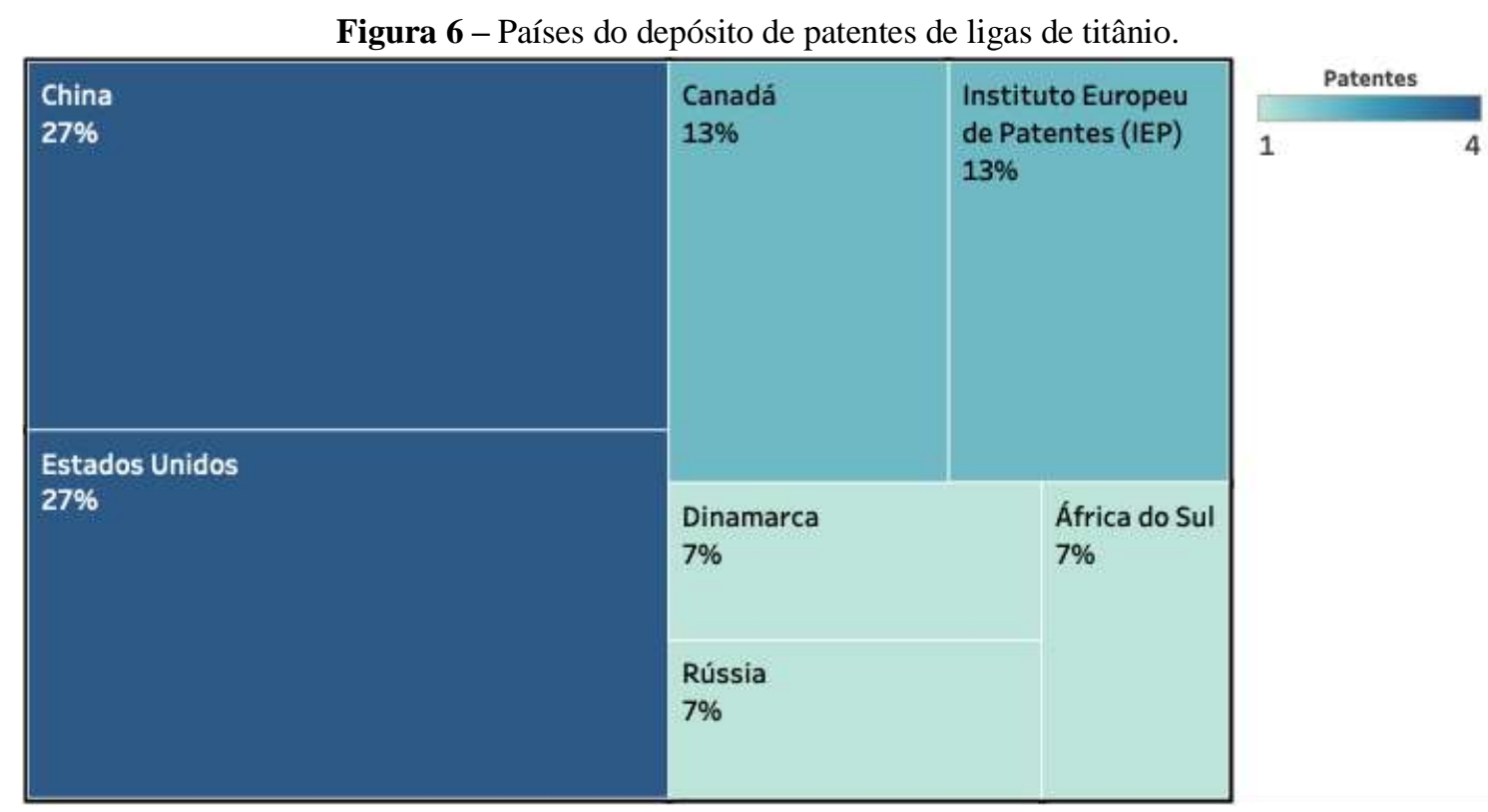

Fonte: Autores (2021)

Outro fato relevante é a comparação da Figura 6 com a Figura 2, produção científica por país, em que se nota que apesar de ser o segundo país que mais publicou artigos científicos sobre ligas de titânio, o Brasil não aparece no ranking de países depositantes de patentes sobre o tema. Desta forma, é importante que haja no país uma estratégia que permita situar o Brasil de forma mais competitiva no que concerne à proteção da propriedade intelectual e transferência destas tecnologias para o mercado, assim como políticas que incentivem e apoie a divulgação destas pesquisas no meio acadêmico.

\section{Considerações Finais}

Este estudo demonstra que a abordagem prospectiva proposta foi eficaz na extração, avaliação, organização e disponibilização de dados sobre pesquisa científica assim como tecnologias patenteadas relacionadas os componentes biomecânicos de titânio ligados a elementos betagênicos aplicados em áreas biomédicas e de saúde.

O número de publicações sobre os componentes biomecânicos de titânio ligados a elementos betagênicos aumentaram significativamente ao longo da linha temporal, especialmente no ano de 2019. Não foi possível afirmar tendência de crescimento para o ano de 2020, uma vez que no momento de realização da busca não foi possível levantar todos os trimestres em razão de limitação atípica provocada pela pandemia do SARS-Cov-2 em todo o mundo.

As revistas ACS Biomaterials Science And Engineering, Materials Science And Engineering C e Materials Science Forum apresentaram o maior número de publicações relacionadas a este tema. Podemos prever que mais estudos sobre componentes biomecânicos de titânio serão publicados nos próximos anos, visto que estes três principais periódicos que foram observados publicações apresentam forte fator de impacto assim como alta pontuação no JCR.

Sobre o Brasil, o estudo demonstra que o país ainda precisa se inserir mais competitivamente nas linhas de P\&D da área de componentes biomecânicos de titânio, necessitando maiores aportes de recursos para o desenvolvimento das pesquisas.

Fundamentado nos resultados apresentados no estudo, é possível compreender que as pesquisas acadêmicas estão alinhadas em estabelecer a fase beta de ligas de titânio em temperaturas de uso para as aplicações biomédicas, bem como, notase uma necessidade maior de alinhamento da rota de processamento termomecânico aos processos das empresas, fator de fundamental relevância para o sucesso mercadológico futuro.

Os resultados deste estudo prospectivo mostraram informações no ambiente nacional relativo às expressivas investigações realizadas a nível de pesquisa acadêmica não transformadas em patentes, confirmando as premissas do estudo de 
(Mueller \& Perucchi, 2014), isto é, que os pesquisadores/inventores, contemporâneos do Brasil encontram cenários de mudanças ideológicas e econômicas, que os pressionam em um caminho diferente da tradição acadêmica. Além disso, é observado asserções relativas a um desajuste na capacidade de coordenação das universidades, mesmo com a criação dos Núcleos de Inovação Tecnológica (s) - NITs. Estas conjecturas, por conseguinte, validam a análise desenvolvida, mesmo tendo em foco o lapso de 6 anos da publicação daquele estudo.

\section{Referências}

ABEC. (2017, 23 de junho). Journal Citation Reports (JCR) Associação Brasileira de Editores Científicos - ABEC. <https://www.abecbrasil.org.br/novo/2017/06/journal-citation-reports-jcr/>.

Almanza, E., Pérez, M. J., Rodríguez, N. A., \& Murr, L. E. (2017). Corrosion resistance of Ti-6Al-4V and ASTM F75 alloys processed by electron beam melting. Journal of Materials Research and Technology, 6(3), 251-257.https://doi.org/10.1016/j.jmrt.2017.05.003

Araújo, C. A. (2006). Bibliometria: evolução histórica e questões atuais. Em questão, 12(1), 11-32.

Chevalier, X., \& Conrozier, T. (2017). Access to highly purified chondroitin sulfate for appropriate treatment of osteoarthritis: a review. Medicine Access@ Point of Care, 1. . (Sage UK: London, England). https://doi.org/10.5301/maapoc.0000022

Fontelo, P., \& Liu, F. (2018). A review of recent publication trends from top publishing countries. Systematic reviews, 7(1), 1-9.

Instituto Nacional de Propriedade Intelectual (2017). Anuário Estatístico de Propriedade Industrial 2000-2016. <http://www.inpi.gov.br/ sobre/estatisticas/arquivos/pagina-inicial/indicadores.zip>.

Kyvik, S., \& Aksnes, D. W. (2015). Explaining the increase in publication productivity among academic staff: A generational perspective. Studies in Higher Education, 40(8), 1438-1453.

Lee, T., Lee, S., Kim, I. S., Moon, Y. H., Kim, H. S., \& Park, C. H. (2020). Breaking the limit of Young's modulus in low-cost Ti-Nb-Zr alloy for biomedical implant applications. Journal of Alloys and Compounds, 828, 154401. https://doi.org/10.1016/j.jallcom.2020.154401

Li, W., Cheng, L., Wang, H., Ning, Y., Jin, C., \& Li, Y. (2020). Bibliometric analysis of patient report outcomes based on CiteSpaceV software. Chinese General Practice, 23 (32), 4128-4134.

Marques, J. P. F. R., \& Marques, R. F. R. (2017). A natureza e o licere de pedido de patente de invenção no Brasil: Concorrência desleal e direito da concorrência em matéria de medicamentos genéricos perante (ameaça de) ajuizamento de ação de infração por parte do requerente de pedido de patente de medicamento de referência. PIDCC: Revista em propriedade intelectual direito contemporâneo, 11(2), 1-61.

Mazieri, M. R., Quoniam, L., \& Santos, A. M. (2016). Inovação a partir das informações de patentes: proposição de modelo Open Source de Extração de Informações de Patentes (Crawler). Revista Gestão \& Tecnologia, 16(1), 76-112.

Mueller, S. P. M., \& Perucchi, V. (2014). Universidades e a produção de patentes: tópicos de interesse para o estudioso da in formação tecnológica. Perspectivas em Ciência da Informação, 19(2), 15-36.

Murillo, A. E., Melo-Maximo, L., Garcia-Farrera, B., Martínez, O. S., Melo-Máximo, D. V., Oliva-Ramirez, J., ... \& Oseguera, J. (2019). Development of AlN thin films for breast cancer acoustic biosensors. Journal of Materials Research and Technology, 8(1), 350-358.

Niinomi, M., Nakai, M., \& Hieda, J. (2012). Development of new metallic alloys for biomedical applications. Acta Biomaterialia, 8(11), 3888-3903. https://doi.org/10.1016/j.actbio.2012.06.037

Nunes, A. R. V., Borborema, S., Araújo, L. S., Malet, L., Dille, J., \& de Almeida, L. H. (2020). Influence of thermo-mechanical processing on structure and mechanical properties of a new metastable $\beta \mathrm{Ti}-29 \mathrm{Nb}-2 \mathrm{Mo}-6 \mathrm{Zr}$ alloy with low Young's modulus. Journal of Alloys and Compounds, 820 , 153078. https://doi.org/10.1016/j.jallcom.2019.153078.

Pereira, A. S., Shitsuka, D. M., Parreira, F. J., \& Shitsuka, R. (2018). Método Qualitativo, quantitativo ou quali-quanti. In Metodologia da Pesquisa Científica. https://repositorio.ufsm.br/bitstream/handle/1/15824/Lic_Computacao_Metodologia-Pesquisa-Cientifica.pdf?sequence=1. Acesso em: 23 fevereiro 2021.

Pires, A. L. R., Bierhalz, A. C., \& Moraes, Â. M. (2015). Biomateriais: tipos, aplicações e mercado. Química nova, 38(7), 957-971.

Prodanov, C. C., \& de Freitas, E. C. (2013). Metodologia do trabalho científico: métodos e técnicas da pesquisa e do trabalho acadêmico-2 a Edição. Editora Feevale.

Pu, Q. H., Lyu, Q. J., \& Su, H. Y. (2016). Bibliometric analysis of scientific publications in transplantation journals from Mainland China, Japan, South Korea and Taiwan between 2006 and 2015. BMJ open, 6(8), e011623.

Souza Neto A. R., Dias, G. F., Silva, R. R., Ramos, A. S. M. (2019). Efeitos dos Softwares de Análise de Dados Qualitativos na Qualidade de Pesquisas. Revista de Administração Contemporânea, 23(3), 373-94.

Quintella, C. M., Meira, M., Kamei, A. G., Tanajura, A. S., \& da Silva, H. R. G. (2011). Prospecção tecnológica como uma ferramenta aplicada em ciência e tecnologia para se chegar à inovação. Revista Virtual de Química, 3(5), 406-415.

Taddei, E. B., Henriques, V. A., Silva, C. R., Cairo, C. A., \& Bottino, M. C. (2007). Ensaio de citotoxicidade e influência do tratamento de solubilização na microestrutura da liga Ti-35Nb-7Zr-5Ta para potenciais aplicações ortopédicas. Matéria (Rio de Janeiro), 12(1), 120 -127. 
Research, Society and Development, v. 10, n. 3, e0810312939, 2021

(CC BY 4.0) | ISSN 2525-3409 | DOI: http://dx.doi.org/10.33448/rsd-v10i3.12939

Teng, C. L., Chew, W. Z., Gupta, E. D., \& Yeap, S. S. (2020). Rheumatological publications from Malaysia: a bibliometric study. Clinical rheumatology, 39(2), $547-552$.

Tubella, F. U. (2019). Z= 22, titanio, Ti. Un metal casi ideal: fuerte, ligero, resistente y biocompatible, pero caro. In Anales de Química. 115(2), 84-84.

Universidade de São Paulo. (2020). Bases para Pesquisa|Escola de Educação Física e Esporte. 〈http://portaleefe.webhostusp.sti.usp.br/bases-para-pesquisa〉. 11 out. 2020 .

World Intellectual Property Organization (2018). Global Innovation Index 2018 (Portuguese Edition): Energizing The World With Innovation. Genebra - Suíça: Autor.

Wu, C. T., Chang, H. T., Wu, C. Y., Chen, S. W., Huang, S. Y., Huang, M., \& Yen, H. W. (2020). Machine learning recommends affordable new Ti alloy with bone-like modulus. Materials Today, 34, 41-50. https://doi.org/10.1016/j.mattod.2019.08.008

Xing, D., Zhao, Y., Dong, S., \& Lin, J. (2018). Global research trends in stem cells for osteoarthritis: a bibliometric and visualized study. International journal of rheumatic diseases, 21(7), 1372-1384.

Xu, W., Lu, X., Tian, J., Huang, C., Chen, M., Yan, Y., \& Wen, C. (2020). Microstructure, wear resistance, and corrosion performance of Ti35Zr28Nb alloy fabricated by powder metallurgy for orthopedic applications. Journal of Materials Science \& Technology, 41, 191-198. https://doi.org/10.1016/j.jmst.2019.08.041 